PROCEEDINGS OF THE

AMERICAN MATHEMATICAL SOCIETY

Volume 135, Number 3, March 2007, Pages 671-676

S 0002-9939(06)08651-5

Article electronically published on August 31, 2006

\title{
CHARACTER DEGREE GRAPHS THAT ARE COMPLETE GRAPHS
}

\author{
MARIAGRAZIA BIANCHI, DAVID CHILLAG, MARK L. LEWIS, \\ AND EMANUELE PACIFICI
}

(Communicated by Jonathan I. Hall)

\begin{abstract}
Let $G$ be a finite group, and write $\operatorname{cd}(G)$ for the set of degrees of irreducible characters of $G$. We define $\Gamma(G)$ to be the graph whose vertex set is $\operatorname{cd}(G)-\{1\}$, and there is an edge between $a$ and $b$ if $(a, b)>1$. We prove that if $\Gamma(G)$ is a complete graph, then $G$ is a solvable group.
\end{abstract}

\section{INTRODUCTION}

Throughout this note, $G$ will be a finite group and $\operatorname{cd}(G)$ will be the set of degrees of irreducible characters of $G$. A theorem of Thompson states that if every degree in $\operatorname{cd}(G)-\{1\}$ is divisible by some prime $p$, then $G$ has a normal $p$-complement (see Corollary 12.2 of 5 or Theorem 23.3 of 4 ). In [2, Berkovich showed that more can be said in this situation. In particular, he proved that if $p$ divides every degree in $\operatorname{cd}(G)-\{1\}$, then $G$ is solvable. In this paper, we will generalize Berkovich's result.

We define $\Gamma(G)$ to be the graph whose vertex set is $\operatorname{cd}(G)-\{1\}$. There is an edge between $a$ and $b$ if $(a, b)>1$. This is the common-divisor character degree graph. If $p$ divides every degree in $\operatorname{cd}(G)-\{1\}$, then $\Gamma(G)$ is a complete graph. We will show that Berkovich's conclusion can be obtained by assuming only that $\Gamma(G)$ is a complete graph.

Main Theorem. If $\Gamma(G)$ is a complete graph, then $G$ is a solvable group.

This result illustrates one difference between the character degree sets of solvable and nonsolvable groups since there exist solvable groups $G$ with $\Gamma(G)$ a complete graph. First, if $G$ is a $p$-group, then $\Gamma(G)$ is a complete graph. Let $p, q$, and $r$ be distinct primes. In Theorem B of [12, Turull constructs for every $p$-group $A$ of order $p^{l}$, a finite $\{q, r\}$-group $H$ and an action of $A$ on $H$ so that $\mathbf{C}_{H}(A)=1$ and the Fitting height of $H$ is $l$. Taking $G$ to be the resulting semi-direct product, it follows from Glauberman correspondence (Theorem 13.1 of [5] or Theorem 18.15 of 4]) that the principal character of $H$ is the only $G$-invariant character in $\operatorname{Irr}(H)$, and it follows that $p$ divides every degree in $\operatorname{cd}(G)$. Finally, the third author, along with Moretó and Wolf, has constructed in [7] for every pair of odd primes $(p, q)$,

Received by the editors October 4, 2005.

2000 Mathematics Subject Classification. Primary 20C15; Secondary 05C25.

(C)2006 American Mathematical Society

Reverts to public domain 28 years from publication 
where $p$ is congruent to 1 modulo 3 and $q$ divides $p+1$, a solvable group $G$ with $\operatorname{cd}(G)=\left\{1,3 q, p^{2} q, 3 p^{3}\right\}$. Taking direct products of $G$ with itself we can get groups with many character degrees that have $\Gamma(G)$ a complete graph, but no prime divides every degree.

To understand the situation when $\Gamma(G)$ is a complete graph and $G$ is a solvable group there seem to be two cases that need to be studied. The first is the case where some prime $p$ divides every degree in $\operatorname{cd}(G)-\{1\}$, and the second is the case where no such prime $p$ exists. We have seen that if $G$ is a group from the first case, then there is no bound on either the derived length or the Fitting height of $G$. On the other hand, Berkovich does obtain some additional information regarding the structure of $G$ in 2. In the case where there is no prime $p$ dividing all the degrees in $\operatorname{cd}(G)-\{1\}$, we have little information since we have few examples.

Looking at the literature, there have been few results about the graph $\Gamma(G)$, and the only result we know of regarding $\Gamma(G)$ when $G$ is nonsolvable is due to McVey. In [9], he proves that if $G$ is a nonsolvable group, and $\Gamma(G)$ is connected, then $\Gamma(G)$ has diameter at most 3 . Notice that our result says in this situation that $\Gamma(G)$ has diameter at least 2. Examples show that both diameters occur among nonsolvable groups.

In the literature, usually another graph $\Delta(G)$ has been attached to $\operatorname{cd}(G)$. This graph takes the primes dividing degrees in $\operatorname{cd}(G)$ to be its vertices, and there is an edge between $p$ and $q$ if $p q$ divides some degree $a \in \operatorname{cd}(G)$. It is not surprising that these two graphs are closely related. It is not difficult to show that one is connected if and only if the other is; and in this case that their diameters differ by at most 1 (see [8]). Recently, it has been shown that $\Delta(G)$ is a complete graph for most simple groups (see [13], 14], and [15]), which perhaps makes it surprising that $\Gamma(G)$ is never a complete graph for a nonsolvable group $G$.

Using tensor induction, the theorem is reduced to the case where $G$ is almost simple. If $G$ is a group of Lie type, the Steinberg character has prime power degree. With the Steinberg character in hand, Thompson's result implies the theorem. The alternating and sporadic cases are handled by explicitly listing two characters of relatively prime degree.

\section{Results}

Before we actually prove the Main Theorem, we must gather some facts. Our proof will be based on the classification of finite simple groups. The main point of the classification is that if $S$ is a nonabelian simple group, then $S$ is either a simple group of Lie type, an alternating group, one of 26 sporadic simple groups, or the Tits group. We gather information on each of these types of simple groups.

We begin with the simple groups of Lie type. For these groups, we look at a particular character called the Steinberg character. The Steinberg character and its properties can be found in many places; we will use Chapter 6 of [3] for our reference. For us, the important property of the Steinberg character is that its degree is a prime power, which is the following result found as Theorem 6.4.7 of [3].

Theorem 1. Let $G$ be a nonabelian simple group of Lie type with defining characteristic $p$. If $\chi$ is the Steinberg character for $G$, then $\chi(1)=|G|_{p}$. 
We also need the following result regarding the Steinberg character that was proved in [10] and [11].

Theorem 2 (Schmid). Let $N$ be a normal subgroup of a group $G$, and suppose that $N$ is isomorphic to a finite simple group of Lie type. If $\theta$ is the Steinberg character for $N$, then $\theta$ extends to $G$.

We next consider the alternating groups. We make the observation that

$$
(n-1)(n-2) / 2=\left(n^{2}-3 n+2\right) / 2=\left(n^{2}-3 n\right) / 2+2 / 2=n(n-3) / 2+1,
$$

and so, the numbers $n(n-3) / 2$ and $(n-1)(n-2) / 2$ are relatively prime.

Theorem 3. If $n \geq 6$, then $\operatorname{Irr}(\operatorname{Alt}(n))$ contains characters of degree $n(n-3) / 2$ and $(n-1)(n-2) / 2$ that extend to $\operatorname{Sym}(n)$.

Proof. Taking into account that $\operatorname{Alt}(n)$ is 4 -transitive for $n$ greater than 5 , we can define two irreducible characters $\sigma$ and $\tau$ for $\operatorname{Alt}(n)$, of degree $n(n-3) / 2$ and $(n-1)(n-2) / 2$ respectively, as in Theorem 11.9 of [4]. It is clear by the definition that both these characters allow an extension to $\operatorname{Sym}(n)$.

For the sporadic groups, the following fact can be found in the atlas, 1]. Often the Tits group, which we denote by ${ }^{2} F_{2}(4)^{\prime}$, is lumped in with the groups of Lie type, and the arguments we use for the groups of Lie type could be adapted for the Tits group. Since the Tits group is in the atlas, it is easier to include it with the sporadic groups. Table 1 will list the atlas characters that we need.

Theorem 4. Let $S$ be a sporadic simple group or the Tits group, and let $A$ be the automorphism group of $S$. Then there exist nonlinear characters $\chi_{m}, \chi_{n} \in \operatorname{Irr}(S)$ so that $\left(\chi_{m}(1), \chi_{n}(1)\right)=1$ and both $\chi_{m}$ and $\chi_{n}$ extend to $A$.

For the last of the preliminary facts, we will need tensor induction of characters. There are several sources for tensor induction. We will be using [6]. Let $H$ be a subgroup of a group $G$, and let $T$ be a right transversal for $H$ in $G$. If $g \in G$ and $t \in T$, then define $t \cdot g$ to be the element of $T$ that lies in $\mathrm{Htg}$. This defines an action of $G$ on $T$. Take $T_{0}$ to be a set of orbit representatives for the action of $\langle g\rangle$ on $T$ via ., and let $s(t)$ denote the size of the $\langle g\rangle$-orbit containing $t$. If $\theta$ is a character of $H$, we define $\theta^{\otimes G}$ as a function of $G$ by $\theta^{\otimes G}(g)=\prod_{t \in T_{0}} \theta\left(t g^{s(t)} t^{-1}\right)$. It is proved in [6] that $\theta^{\otimes G}$ is a character. In addition, if $N$ is a normal subgroup of $G$ so that $N \subseteq H$, then it is proved in Lemma 4.1 of [6] for $n \in N$ that $\theta^{\otimes G}(n)=\prod_{t \in T} \theta\left(t n t^{-1}\right)$. We note that tensor induction is also described in Theorem 25.3 of 4 .

Using tensor induction, we prove the following lemma. This lemma should be compared with Lemma 25.5 of [4].

Lemma 5. Let $N$ be a minimal normal subgroup of $G$ so that $N=S_{1} \times \cdots \times S_{t}$, where $S_{i} \cong S$, a nonabelian simple group. Let $A$ be the automorphism group of $S$. If $\sigma \in \operatorname{Irr}(S)$ extends to $A$, then $\sigma \times \cdots \times \sigma \in \operatorname{Irr}(N)$ extends to $G$.

Proof. Let $H=\mathbf{N}_{G}\left(S_{1}\right)$ and $C=\mathbf{C}_{G}\left(S_{1}\right)$. We know that $G$ acts transitively on $\left\{S_{1}, \ldots, S_{t}\right\}$, so $|G: H|=t$. Take $T$ to be a right transversal for $H$ in $G$, and label the elements in $T=\left\{x_{1}, \ldots, x_{t}\right\}$ so that $S_{1}^{x_{i}}=S_{i}$. Let $\sigma_{i} \in \operatorname{Irr}(N)$ be the 
TABle 1. Degrees of Sporadic Groups and the Tits Group

\begin{tabular}{|c|c|c|c|c|c|}
\hline Group & Chars. & Degrees & Group & Chars. & Degrees \\
\hline$M_{11}$ & $\begin{array}{l}\chi_{2} \\
\chi_{5}\end{array}$ & $\begin{array}{l}10=2 \cdot 5 \\
11\end{array}$ & $O^{\prime} N$ & $\begin{array}{c}\chi_{2} \\
\chi_{19}\end{array}$ & $\begin{array}{l}10944=2^{6} \cdot 3^{2} \cdot 19 \\
116963=7^{3} \cdot 11 \cdot 31\end{array}$ \\
\hline$M_{12}$ & $\begin{array}{l}\chi_{7} \\
\chi_{8}\end{array}$ & $\begin{array}{l}54=2 \cdot 3^{3} \\
55=5 \cdot 11\end{array}$ & $\mathrm{Co}_{3}$ & $\begin{array}{l}\chi_{2} \\
\chi_{5}\end{array}$ & $\begin{array}{l}23 \\
275=5^{2} \cdot 11\end{array}$ \\
\hline$J_{1}$ & $\begin{array}{l}\chi_{4} \\
\chi_{6}\end{array}$ & $\begin{array}{l}76=2^{2} \cdot 19 \\
77=7 \cdot 11\end{array}$ & $\mathrm{Co}_{2}$ & $\begin{array}{l}\chi_{2} \\
\chi_{4}\end{array}$ & $\begin{array}{l}23 \\
275=5^{2} \cdot 11\end{array}$ \\
\hline$M_{22}$ & $\begin{array}{l}\chi_{2} \\
\chi_{5}\end{array}$ & $\begin{array}{l}21=3 \cdot 7 \\
55=5 \cdot 11\end{array}$ & $F i_{22}$ & $\begin{array}{l}\chi_{56} \\
\chi_{57}\end{array}$ & $\begin{array}{l}1441792=2^{17} \cdot 11 \\
1791153=3^{9} \cdot 7 \cdot 13\end{array}$ \\
\hline$J_{2}$ & $\begin{array}{c}\chi_{6} \\
\chi_{13}\end{array}$ & $\begin{array}{l}36=2^{2} \cdot 3^{2} \\
175=5^{2} \cdot 7\end{array}$ & $H N$ & $\begin{array}{l}\chi_{10} \\
\chi_{45}\end{array}$ & $\begin{array}{l}16929=3^{4} \cdot 11 \cdot 19 \\
3200000=2^{10} \cdot 5^{5}\end{array}$ \\
\hline$M_{23}$ & $\begin{array}{l}\chi_{2} \\
\chi_{3}\end{array}$ & $\begin{array}{l}22=2 \cdot 11 \\
45=3^{2} \cdot 5\end{array}$ & $L y$ & $\begin{array}{c}\chi_{7} \\
\chi_{50}\end{array}$ & $\begin{array}{l}120064=2^{8} \cdot 7 \cdot 67 \\
53765625=3 \cdot 5^{6} \cdot 31 \cdot 37\end{array}$ \\
\hline${ }^{2} F_{4}(2)^{\prime}$ & $\begin{array}{l}\chi_{4} \\
\chi_{8}\end{array}$ & $\begin{array}{l}27=3^{3} \\
325=5^{2} \cdot 13\end{array}$ & $T h$ & $\begin{array}{l}\chi_{2} \\
\chi_{7}\end{array}$ & $\begin{array}{l}248=2^{3} \cdot 31 \\
30875=5^{3} \cdot 13 \cdot 19\end{array}$ \\
\hline$H S$ & $\begin{array}{l}\chi_{2} \\
\chi_{7}\end{array}$ & $\begin{array}{l}22=2 \cdot 11 \\
175=5^{2} \cdot 7\end{array}$ & $F i_{23}$ & $\begin{array}{c}\chi_{4} \\
\chi_{94}\end{array}$ & $\begin{array}{l}5083=13 \cdot 17 \cdot 23 \\
504627200=2^{18} \cdot 5^{2} \cdot 7 \cdot 11\end{array}$ \\
\hline$J_{3}$ & $\begin{array}{c}\chi_{6} \\
\chi_{13}\end{array}$ & $\begin{array}{l}324=2^{3} \cdot 3^{4} \\
1615=5 \cdot 17 \cdot 19\end{array}$ & $\mathrm{Co}_{1}$ & $\begin{array}{c}\chi_{3} \\
\chi_{17}\end{array}$ & $\begin{array}{l}299=13 \cdot 23 \\
673750=2 \cdot 5^{4} \cdot 7^{2} \cdot 11\end{array}$ \\
\hline$M_{24}$ & $\begin{array}{l}\chi_{2} \\
\chi_{3}\end{array}$ & $\begin{array}{l}23 \\
45\end{array}=3^{2} \cdot 5$ & $J_{4}$ & $\begin{array}{c}\chi_{2} \\
\chi_{11}\end{array}$ & $\begin{array}{l}1333=31 \cdot 43 \\
1776888=2^{3} \cdot 3^{2} \cdot 23 \cdot 29 \cdot 37\end{array}$ \\
\hline$M^{c} L$ & $\begin{array}{c}\chi_{2} \\
\chi_{14}\end{array}$ & $\begin{array}{l}22=2 \cdot 11 \\
5103=3^{6} \cdot 7\end{array}$ & $F i_{24}^{\prime}$ & $\begin{array}{l}\chi_{2} \\
\chi_{6}\end{array}$ & $\begin{array}{l}8671=23 \cdot 29 \cdot 13 \\
1603525=5^{2} \cdot 7^{3} \cdot 11 \cdot 17\end{array}$ \\
\hline $\mathrm{He}$ & $\begin{array}{c}\chi_{9} \\
\chi_{15}\end{array}$ & $\begin{array}{l}1275=3 \cdot 5^{2} \cdot 17 \\
6272=2^{7} \cdot 7^{2}\end{array}$ & $B$ & $\begin{array}{c}\chi_{2} \\
\chi_{119}\end{array}$ & $\begin{array}{l}4371=3 \cdot 31 \cdot 47 \\
2642676197359616 \\
\quad=2^{39} \cdot 11 \cdot 19 \cdot 23\end{array}$ \\
\hline Suz & $\begin{array}{c}\chi_{5} \\
\chi_{20}\end{array}$ & $\begin{array}{l}783=3^{3} \cdot 29 \\
45500=2^{2} \cdot 5^{3} \cdot 7 \cdot 13 \\
143=11 \cdot 13 \\
248832=2^{10} \cdot 3^{5}\end{array}$ & $M$ & $\begin{array}{c}\chi_{2} \\
\chi_{16}\end{array}$ & $\begin{array}{l}196883=59 \cdot 71 \cdot 47 \\
8980616927734375 \\
\quad=5^{9} \cdot 7^{6} \cdot 11^{2} \cdot 17 \cdot 19\end{array}$ \\
\hline
\end{tabular}

character whose $i$ th component is $\sigma$, and the other components are $1_{S}$. Observe that $\sigma_{1}^{x_{i}}=\sigma_{i}$. Also,

$$
\prod_{i=1}^{t} \sigma_{i}=\sigma \times \cdots \times \sigma \in \operatorname{Irr}(N) .
$$

We know that $H / C$ is isomorphic to a subgroup of the automorphism group of $S_{1}$, and so, $H / C$ is isomorphic to a subgroup of $A$. Also, we know that $S_{1} \cap C=$ $\mathbf{Z}\left(S_{1}\right)=1$, so $S_{1} C=S_{1} \times C \subseteq H$. Observe that $N \cap C=S_{2} \times \cdots \times S_{t}$, so $\sigma_{1}$ extends to $\sigma \times 1_{C} \in \operatorname{Irr}\left(S_{1} C\right)$. Now, we know that $\sigma \times 1_{C}$ viewed as a character 
of $S_{1} C / C \cong S_{1}$ extends to the automorphism group of $S_{1}$, so $\sigma \times 1_{C}$ extends to $\theta \in \operatorname{Irr}(H / C)$. We let $\chi=\theta^{\otimes G}$.

We will show that $\chi$ is an extension of $\sigma \times \cdots \times \sigma$. Given an element $n \in N$, we have

$$
\begin{aligned}
\chi(n) & =\theta^{\otimes G}(n)=\prod_{i=1}^{t} \theta\left(x_{i} n x_{i}^{-1}\right)=\prod_{i=1}^{t} \sigma_{1}\left(x_{i} n x_{i}^{-1}\right) \\
& =\prod_{i=1}^{t} \sigma_{1}^{x_{i}}(n)=\prod_{i=1}^{t} \sigma_{i}(n)=(\sigma \times \cdots \times \sigma)(n) .
\end{aligned}
$$

It follows that $\chi_{N}=\sigma \times \cdots \times \sigma$ as desired.

We now are ready to prove the Main Theorem.

Proof of the Main Theorem. We will prove the contrapositive. In other words, we assume that $G$ is not solvable, and we show that $\Gamma(G)$ is not a complete graph. Since $G$ is not solvable, we can find normal subgroups $M$ and $N$ in $G$ so that $N / M$ is a nonabelian chief factor for $G$. Given elements $a, b \in \operatorname{cd}(G / M)$, it is easy to see that $a$ and $b$ are adjacent in $\Gamma(G / M)$ if and only if they are adjacent in $\Gamma(G)$. Thus, it suffices to show that $\Gamma(G / M)$ is not a complete graph, and so, we may assume that $M=1$.

Now, $N$ is a nonabelian minimal normal subgroup of $G$, so $N=S_{1} \times \cdots \times S_{t}$, where $S_{i} \cong S$ for some nonabelian simple group $S$. First, suppose that $S$ is a simple group of Lie type with characteristic $p$ for some prime $p$. Take $\sigma$ to be the Steinberg character of $S$ so $\sigma(1)$ is a power of $p$. In light of Theorem 2, we can use Lemma 5 to see that $(\sigma(1))^{t}$ lies in $\operatorname{cd}(G)$. Since $G$ does not have a normal $p$-complement, we can use Thompson's theorem (Corollary 12.2 of [5]) to see that $\operatorname{cd}(G)-\{1\}$ must have a degree $a$ that is not divisible by $p$. Hence, $(\sigma(1))^{t}$ and $a$ are relatively prime, so $\Gamma(G)$ is not a complete graph.

Suppose that $S$ is an alternating group on $n$ items with $n \geq 7$ or $S$ is a sporadic simple group or the Tits group. (Note that $\mathrm{Alt}(5) \cong \mathrm{PSL}_{2}(4) \cong \mathrm{PSL}_{2}(5)$ and $\operatorname{Alt}(6) \cong \mathrm{PSL}_{2}(9)$, so these two cases have already been handled.) For $n \geq 7$, we know that the automorphism group of $\operatorname{Alt}(n)$ is $\operatorname{Sym}(n)$. Let $A$ be the automorphism group of $S$. We use Theorem 3 or Theorem 4 to find nonlinear characters $\sigma, \tau \in \operatorname{Irr}(S)$ so that $(\sigma(1), \tau(1))=1$ and $\sigma$ and $\tau$ both extend to $A$. Thus, we may use Lemma 5 to see that $(\sigma(1))^{t}$ and $(\tau(1))^{t}$ both lie in $\operatorname{cd}(G)$. Obviously, these degrees are relatively prime, so $\Gamma(G)$ is not a complete graph.

\section{REFERENCES}

[1] J. H. Conway, R. T. Curtis, S. P. Norton, R. A. Parker, and R. A. Wilson, "Atlas of Finite Groups," Oxford University Press, London, 1984. MR0827219 (88g:20025)

[2] Y. Berkovich, Finite groups with small sums of degrees of some non-linear irreducible characters, J. Algebra 171 (1995), 426-443. MR1315905 (96c:20015)

[3] R. W. Carter "Finite Groups of Lie Type," Wiley, New York, 1985. MR0794307 (87d:20060)

[4] B. Huppent, "Character Theory of Finite Groups," Walter DeGruyter, Berlin, 1998. MR 1645304 (99j:20011)

[5] I. M. IsAACS, "Character Theory of Finite Groups," Academic Press, San Diego, 1976. MR0460423 (57:417)

[6] I. M. IsAACS, Character correspondences in solvable groups, Adv. in Math. 43 (1982), 284306. MR0648802 (83i:20007) 
[7] M. L. Lewis, A. Moretó, And T. R. Wolf, Non-divisibility among character degrees, J. Group Theory 8 (2005), 561-588. MR2165291

[8] M. L. LEwIS, An overview of graphs associated with character degrees and conjugacy class sizes in finite groups, to appear in Rocky Mountain J. Math.

[9] J. K. MCVey, Bounding graph diameters of nonsolvable groups, J. Algebra 282 (2004), 260-277. MR2097583 (2005h:20011)

[10] P. Schmid, Rational matrix groups of a special type, Linear Algebra Appl. 71 (1985), 289-293. MR0813053 (87e:20018)

[11] P. Schmid, Extending the Steinberg representation, J. Algebra 150 (1992), 254-256. MR.1174899 (93k:20016)

[12] A. Turull, Generic fixed point free action of arbitrary finite groups, Math. Z. 187 (1984), 491-503. MR0760049 (86a:20020)

[13] D. L. White, Degree graphs of simple groups of exceptional Lie type, Comm. Algebra 32 (2004), 3641-3649. MR2097483 (2005h:20022)

[14] D. L. White, Degree graphs of simple linear and unitary groups, to appear in Comm. Algebra.

[15] D. L. White, Degree graphs of simple orthogonal and symplectic groups, to appear in $J$. Algebra.

Dipartimento di Matematica "F. Enriques", Università Degli Studi Di Milano, Via C. SAldini 50, 20133 Milano, Italy

E-mail address: Mariagrazia.Bianchi@mat.unimi.it

Department of Mathematics, Technion, Israel Institute of Technology, Haifa 32000, ISRAEL

E-mail address: chillag@techunix.technion.ac.il

Department of Mathematical Sciences, Kent State University, Kent, Ohio 44242

E-mail address: lewis@math.kent.edu

Dipartimento di Matematica "F. Enriques", Università Degli Studi Di Milano, Via C. Saldini 50, 20133 Milano, Italy

E-mail address: Emanuele.Pacifici@mat.unimi.it 